$06.1 ; 13.1$

\title{
Спин-волновой резонанс в синтетическом антиферромагнетике $\left[\left(\mathrm{Co}_{0.88} \mathrm{Fe}_{\mathbf{0 . 1 2}}\right) / \mathrm{Cu}\right]_{N}$
}

\author{
(C) И.Г. Важенина ${ }^{1}$, Р.С. Исхаков ${ }^{1}$, М.А. Миляев ${ }^{2}$, Л.И. Наумова ${ }^{2}$, М.В. Рауцкий ${ }^{1}$ \\ ${ }^{1}$ Институт физики им. Л.В. Киренского СО РАН, ФИЦ КНЦ СО РАН, Красноярск, Россия \\ ${ }^{2}$ Институт физики металлов им. М.Н. Михеева УрО РАН, Екатеринбург, Россия \\ E-mail: irina-vazhenina@mail.ru
}

Поступило в Редакцию 26 июня 2020г.

В окончательной редакции 27 июля 2020 r.

Принято к публикации 27 июля 2020 г.

\begin{abstract}
Методом спин-волнового резонанса исследован синтетический антиферромагнетик $\left[\left(\mathrm{Co}_{0.88} \mathrm{Fe}_{0.12}\right) / \mathrm{Cu}\right]_{N}$ во всем диапазоне углов внешнего постоянного магнитного поля относительно нормали к поверхности пленки. Исследования демонстрируют, что рассматриваемая сверхрешетка представляет собой две обменно-связанные магнитные подсистемы, каждая из которых проявляется в регистрируемых спектрах в виде серий спинволновых мод. Установлена зависимость величины линейной области распространения стоячей спиновой волны от ориентации образца во внешнем магнитном поле.
\end{abstract}

Ключевые слова: спин-волновой резонанс, синтетический антиферромагнетик, константа обменного взаимодействия.

DOI: 10.21883/PJTF.2020.21.50193.18433

Сверхрешетки $\left[\mathrm{Co}_{1-x} \mathrm{Fe}_{x} / \mathrm{Cu}\right]_{N}$ обладают уникальным сочетанием определенных магнитных свойств, которое позволяет использовать их в магнитных сенсорах с рекордными техническими параметрами [1-3]. Результаты исследований микроволнового гигантского магниторезистивного эффекта ( $\mu \mathrm{GMR})[1-3]$ демонстрируют эффективность электродинамических методик сантиметрового диапазона длин волн для описания электромагнитных свойств сверхрешеток.

Традиционными методами измерения динамических характеристик магнитных систем являются методы ферромагнитного (ФМР) и спин-волнового резонанса (СВР) [4-6]. Угловые зависимости спектров СВР и ФМР позволяют определить такие параметры магнитной системы, как эффективная намагниченность $M_{e f f}$, константа обменного взаимодействия $A$, константы ортогональной анизотропии и анизотропии в плоскости пленки, константа поверхностной анизотропии.

Результаты теоретических $[7,8]$ и экспериментальных $[9,10]$ исследований динамических характеристик мультислойных структур демонстрируют зависимость формы спектра как от структурных (толщины слоев и их количества), так и от магнитных (величины намагниченности, поля анизотропии) параметров отдельного слоя [11]. Таким образом, методы ФМР и СВР позволят установить интегральные магнитные параметры планарной сверхрешетки $[\mathrm{CoFe} / \mathrm{Cu}]$ и оценить их взаимосвязь с другими эффектами, наблюдаемыми на данных композитах [12].

Исследованы динамические свойства сверхрешетки $\left[\left(\mathrm{Co}_{0.88} \mathrm{Fe}_{0.12}\right) / \mathrm{Cu}\right]_{N}$ с толщиной немагнитной прослойки $t_{s}=0.95 \mathrm{~nm}$. Образец синтезировался методом магнетронного напыления с использованием установки MPS-4000-C6 [13]. В качестве подло- жек использовалось стекло Corning. Образец представлял собой планарную наноструктуру с композицией $\mathrm{Ta}(5 \mathrm{~nm}) / \mathrm{Ni}_{48} \mathrm{Fe}_{12} \mathrm{Cr}_{40}(5 \mathrm{~nm}) /\left[\mathrm{Co}_{88} \mathrm{Fe}_{12}(1.5 \mathrm{~nm}) /\right.$ $\mathrm{Cu}(0.95 \mathrm{~nm})]_{24} / \mathrm{Ta}(5 \mathrm{~nm})$.

Рентгеновские исследования на дифрактометре ДРОН-3М с использованием $\mathrm{CoK}_{\alpha}$-излучения показали, что сверхрешетка обладает совершенной структурой слоев с ГЦК-решеткой и аксиальной текстурой $\langle 111\rangle$ с осью, нормальной к плоскости слоев. Результаты измерения микроволнового GMR исследуемой структуры представлены в [3]. Согласно зависимостям $M(H)$, измеренным для подобных структур [13], величина поля насыщения принимает значения в диапазоне 200-300 Ое при различных составах буферного слоя.

Измерения СВЧ-спектров пленок было выполнено на оборудовании КРЦКП ФИЦ КНЦ СО РАН (спектрометре ELEXSYS E580 Bruker, Германия) при комнатной температуре в $X$-диапазоне (частота накачки резонатора $f=9.47 \mathrm{GHz}$ ), образец помещался в пучность переменного магнитного поля $h_{\sim}$ проходного резонатора. Постоянное магнитное поле Н изменялось как в плоскости пленки по углу $\varphi$, так и в плоскости, параллельной нормали пленки, при различных значениях угла $\theta$, который отсчитывался от нормали к плоскости пленки.

Зависимость положений резонансных полей $H_{n}$ от волнового вектора $k$, который связан с номером моды $n$ выражением $k=n \pi / d(d-$ толщина пленки, $n$ принимает значения $1,3,5, \ldots$ для объемных спиновых мод и $n=0$ для однородной моды ФМР), при $\theta=90^{\circ}$ имеет вид [14]:

$$
H_{n}=\left\{\left[\left(\frac{\omega}{\gamma}\right)^{2}+\left(2 \pi M_{e f f}\right)^{2}\right]^{1 / 2}-2 \pi M_{e f f}\right\}-\eta_{e f f} k_{n}^{2} .
$$



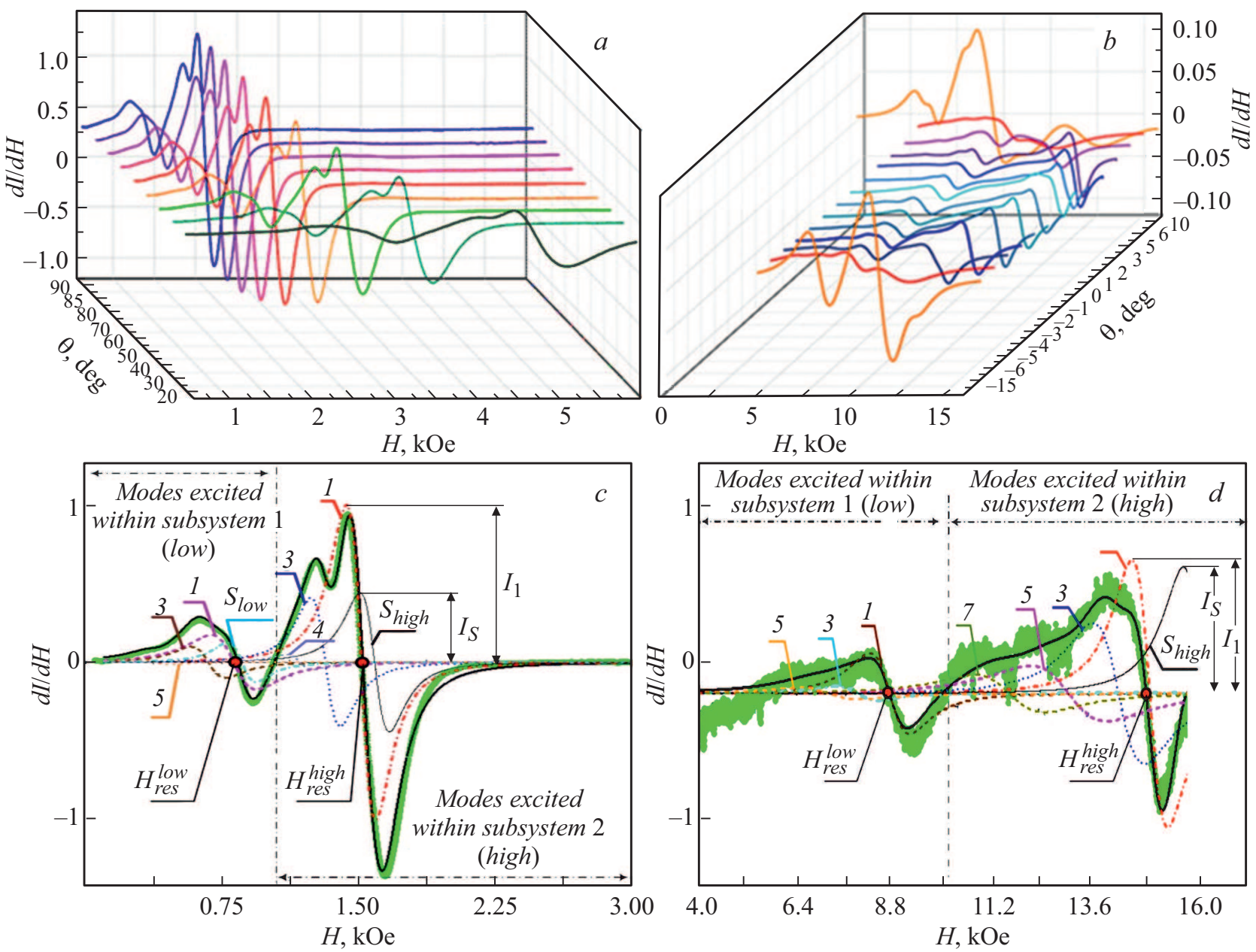

Pис. 1. Эволюция спектров в диапазоне углов от 90 до $20^{\circ}(a)$ и от 10 до $-15^{\circ}(b)$, а также пример разложения экспериментальных спектров при $\theta=90(c)$ и $0^{\circ}(d)$. Буквой $S$ обозначены поверхностные моды, буквой $I-$ интенсивности поверхностной и первой объемной мод. Линиями (на частях $c$ и $d$ ), обозначенными арабскими цифрами, отмечены объемные стоячие спиновые моды.

Позиции резонансных полей $H_{n}$ при $\theta=0^{\circ}$ определяются как [15]:

$$
H_{n}=\frac{\omega}{\gamma}+4 \pi M_{e f f}-\eta_{e f f} k_{n}^{2}
$$

где $\omega=2 \pi f-$ циклическая частота $\left[s^{-1}\right]$, $\gamma=1.758 \cdot 10^{7}[\mathrm{~Hz} / \mathrm{Oe}]-$ гиромагнитное соотношение, $\eta_{\text {eff }}=2 A / M_{s}$ - спин-волновая жесткость $\left[\mathrm{Oe} \cdot \mathrm{cm}^{2}\right]$, связанная с константой обменного взаимодействия $A[\mathrm{erg} / \mathrm{cm}], M_{S}$ - намагниченность насыщения.

Вне зависимости от геометрии эксперимента СВР $\left(\theta=0\right.$ или $\left.90^{\circ}\right)$ эффективная обменная жесткость в полевых координатах вычисляется по формуле

$$
\tilde{\eta}_{e f f}=\frac{H_{1}-H_{n}}{n^{2}-1}
$$

Вклад анизотропии можно оценить из уравнений ФМР для магнитно-анизотропных материалов, полученных в приближении эффективных полей [15], для $\theta=90^{\circ}$

$$
\frac{\omega}{\gamma}=\left[\left(H+H_{e f f}\right)\left(H+H_{u}\right)\right]^{1 / 2}
$$

и для $\theta=0^{\circ}$

$$
\frac{\omega}{\gamma}=\left[\left(H-H_{e f f}\right)\left(H-H_{e f f}-H_{u}\right)\right]^{1 / 2} .
$$

Здесь $H_{e f f}=4 \pi M_{S}+H_{K}-$ эффективное поле, учитывающее влияние упругих напряжений, $H_{u}-$ поле одноосной анизотропии.

Кривые СВЧ-поглощения синтетического антиферромагнетика $[\mathrm{CoFe} / \mathrm{Cu}]_{N}$ при ориентации поля в плоскости пленки $\left(\theta=90^{\circ}\right)$ демонстрируют сложный спектр с неизменной структурой (одинаковые значения резонансного поля и ширины линии отдельных составляющих спектра) во всем диапазоне углов $\varphi$, что свидетельствует об изотропности магнитных параметров в плоскости пленки. Кривые СВЧ-поглощения, когда внешнее постоянное магнитное поле $H$ изменяется относительно нормали к плоскости пленки, имеют вид сложного составного спектра во всем диапазоне углов $\theta$ (рис. $1, a$ и $b$ ). Экспериментальные СВЧ-спектры были разложены на составляющие с помощью дифференцированных функций Лоренца (см. пример на рис. $1, c$ и $d$ ). 

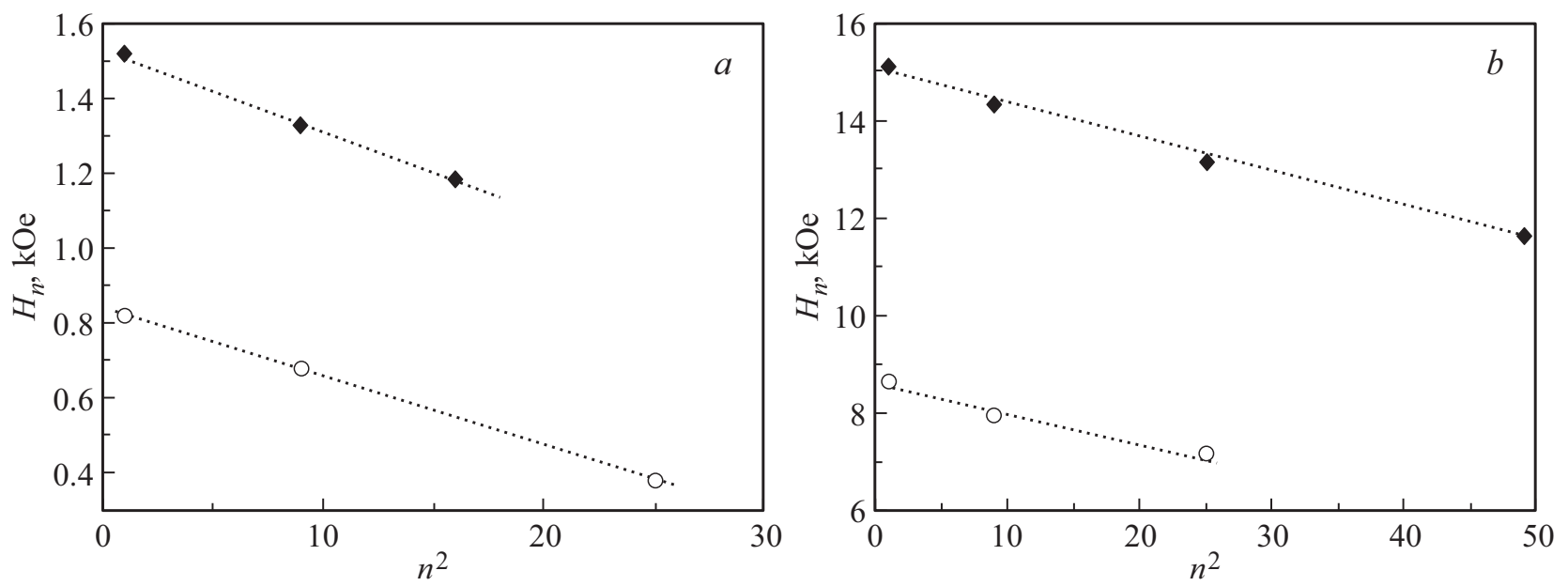

Рис. 2. Экспериментальные дисперсионные зависимости $H_{n}\left(n^{2}\right)$ для случая $\theta=90(a)$ и $0^{\circ}(b)$. Ромбами отмечены значения для высокополевой части спектра, кружками - для низкополевой части спектра.

Анализ спектров позволяет сделать следующие выводы: во всем измеряемом диапазоне углов в пленке возбуждаются как стоячие объемные спиновые моды, так и поверхностные; спектр СВЧ при любом угле $\theta$ состоит из двух частей: низкополевой и высокополевой. Наличие в спектрах СВР двух частей, описываемых отдельными сериями объемных стоячих спиновых мод, дает основание рассматривать сверхрешетку как две магнитные подсистемы с различными величинами магнитных параметров. Способность методов ФМР и СВР фиксировать двухфазность продемонстрирована в $[14,16]$.

Аппроксимация экспериментальных зависимостей $H_{n}\left(n^{2}\right)$ при $\theta=90$ и $0^{\circ}$ (рис. 2) позволяет определить величины резонансных полей ФМР $(n=0)$, а выражения (4) и (5) позволяют оценить $M_{S}$ и $H_{u}$ для каждой подсистемы (см. таблицу).

С помощью (3) и зависимостей $H_{n}\left(n^{2}\right)$ (рис. 2) были рассчитаны величины эффективной обменной жесткости $\tilde{\eta}_{e f f}: \tilde{\eta}_{e f f}^{\perp} \approx 80$ Ое для обеих подсистем в случае $\theta=0^{\circ}$ и $\tilde{\eta}_{e f f}^{\|} \approx 20$ Ое для обеих подсистем в случае $\theta=90^{\circ}$. В то же время известно, что в однородных пленках экспериментальные значения $\eta_{\text {eff }}$ в обеих геометриях получаются равными [14]. Разницу в экспериментальных значениях $\tilde{\eta}_{e f f}$ можно объяснить изменением линейного размера планарной области, в которой происходит возбуждение стоячей спиновой волны, т. е. некой эффективной толщины подсистемы $d_{e f f}^{i}$. Последнее указывает, что для $\theta=0$ и $90^{\circ}$ эффективное закрепление обменной спиновой волны осуществляется внутри пленки, но при различных толщинах $d_{e f f}^{i}$. В [17] обсуждалась возможность формирования узлов обменной спиновой волны внутри мультислойной пленки внутренними граничными условиями, а не традиционными киттелевскими граничными условиями на внешних поверхностях пленки. Особенности спектров СВР в двухслойных пленках, вызванные смещением узла стоячей спиновой волны с межслойной границы, экспериментально регистрировались в работе [18]. Мы предполагаем, что величина $d_{e f f}^{i}$ в различных геометриях меняется вдвое, что следует из экспериментально обнаруженного соотношения $\left(\tilde{\eta}_{e}{ }^{\perp} f f / \tilde{\eta}_{e f f}\right)=4$ (см. дополнительные материалы в онлайн-версии статьи).

Предполагая, что при $\theta=90^{\circ} d_{e f f}^{i}=58.8 \mathrm{~nm}$, и используя величину намагниченности насыщения $M_{S h i g h}$ из таблицы и выражение (3), получим величину эффективной константы обменного взаимодействия $A_{e f f} \sim 0.4 \cdot 10^{-7} \mathrm{erg} / \mathrm{cm}$. Заметим, что величина $A_{\text {eff }}$ для пленок ГЦК-Со $(200 \mathrm{~nm})$ оценивается как $1.3 \cdot 10^{-6} \mathrm{erg} / \mathrm{cm}[10]$; при уменьшении толщины Со величина $A_{e f f}$ может уменьшиться на порядок [19]. Сильное отличие $A_{e f f}$ для мультислойных систем с ультратонким слоем $\mathrm{CoFe}(\sim 1.5 \mathrm{~nm})$ при толщине немагнитной прослойкой $\mathrm{Cu} \sim 0.95 \mathrm{~nm}$ от аналогичного параметра однослойных пленок объясняется интегральным вкладом в данный параметр парциальных обменных взаимодействий отдельных ферромагнитных слоев и парциального обмена между ферромагнитными слоями через немагнитную прослойку.

Магнитные параметры

\begin{tabular}{|c|c|c|c|}
\hline \multicolumn{2}{|c|}{ Магнитные параметры } & Подсистема 1 & Подсистема 2 \\
\hline $\begin{array}{l}\text { Резонансные } \\
\text { поля ФМР } \\
\text { Намагниченн } \\
\text { Поле одноосг }\end{array}$ & $\begin{aligned} \theta & =90^{\circ} \\
\theta & =0^{\circ}\end{aligned}$ & $\begin{aligned} H_{0 \text { low }}^{\|} & =840 \mathrm{Oe} \\
H_{0 \text { low }}^{\perp} & =8700 \mathrm{Oe} \\
M_{\text {Slow }} & =870 \mathrm{G} \\
H_{\text {ulow }} & \approx 3 \mathrm{Oe}\end{aligned}$ & $\begin{aligned} H_{0 \text { high }}^{\|} & =1550 \mathrm{Oe} \\
H_{0 \text { high }}^{\perp} & =15100 \mathrm{Oe} \\
M_{S \text { high }} & =939 \mathrm{G} \\
H_{\text {u high }} & \cong-730 \mathrm{Oe}\end{aligned}$ \\
\hline
\end{tabular}


Сверхрешетка $\left[\left(\mathrm{Co}_{0.88} \mathrm{Fe}_{0.12}\right) / \mathrm{Cu}\right]_{N}$ толщиной $\sim 60 \mathrm{~nm}$ (в отличие от аналогичной системы толщиной $\sim 30 \mathrm{~nm}$ ) представляет собой две обменно-связанные магнитные подсистемы, которые проявляются в виде двух наборов спиновых обменных стоячих мод в спектре СВР во всем диапазоне угла $\theta$. Подсистемы обладают разными по знаку и величине одноосными перпендикулярными константами анизотропии. Обнаружено изменение величины линейной области распространения обменной спиновой волны в сверхрешетке в зависимости от ориентации пленки во внешнем постоянном магнитном поле. Выявленные особенности магнитной системы могут стать механизмом для управления величиной GMR при синтезе сверхрешеток.

\section{Финансирование работы}

Работа выполнена в рамках государственного задания Министерства образования и науки РФ (тема „Спин“ № AААА-A18-118020290104-2) при частичной поддержке Российского фонда фундаментальных исследований (проект № 20-42-660018).

\section{Конфликт интересов}

Авторы заявляют, что у них нет конфликта интересов.

\section{Список литературы}

[1] Ринкевич А.Б., Ромашев Л.Н., Устинов В.В. // ЖЭТФ. 2000. T. 117. B. 5. C. $960-968$.

[2] Kuanr B.K., Kuanr A.V., Grünberg P., Nimtz G. // Phys. Lett. A. 1996. V. 221. P. 245-252.

[3] Ринкевич А.Б., Пахомов Я.А., Кузнецов Е.А., Клепикова А.С., Миляев М.А., Наумова Л.И., Устинов В.В. // Письма в ЖТФ. 2019. Т. 45. В. 5. С. 42-44.

[4] Гуревич А.Г. Магнитный резонанс в ферритах и антиферромагнетиках. М.: Наука, 1973. $591 \mathrm{c}$

[5] Ферромагнитный резонанс / Под ред. С.В. Вонсовский. М.: Физматгиз, 1961. $343 \mathrm{c}$.

[6] Саланский Н.М., Ерухимов М.Ш. Физические свойства и применение магнитных пленок. Новосибирск: Наука, 1975. $221 \mathrm{c}$.

[7] Ignatchenko V.A., Mankov Y.I., Maradudin A.A. // Phys. Rev. B. 2000. V. 62. P. 2181-2184.

[8] Kruglyak V.V., Kuchko A.N. // Physica B. 2003. V. 339. P. 130 133.

[9] Исхаков Р.С., Столяр С.В., Чеканова Л.А., Чижик М.В. // ФТТ. 2012. Т. 54. В. 4. С. 704-708. http://journals.ioffe.ru/articles/580

[10] Исхаков Р.С., Мороз Ж.М., Чеканова Л.А., Шалыгина E.E., Wenema H.A. // ФТТ. 2003. Т. 45. В. 5. C. 846-851. http://journals.ioffe.ru/articles/4617

[11] Khodadadi B., Mohammadi J.B., Jones J.M., Srivastava A., Mewes C., Mewes T., Kaiser C. // Phys. Rev. Appl. 2017. V. 8. P. 014024.

[12] Устинов В.В., Ринкевич А.Б., Важсенина И.Г., Миляев М.А. // ЖЭТФ. 2020. Т. 158. В. 1. С. 139-150. https://doi.org/: 10.31857/S0044451020070135
[13] Банникова Н.С., Миляев М.А., Наумова Л.И., Проглядо В.В., Кринищина Т.П., Чернышева Т.А., Устинов В.В. // ФММ. 2015. Т. 116. № 2. C. 165-170.

[14] Исхаков Р.С., Середкин В.А., Чеканова Л.А., Яковчук В.Ю. // Письма в ЖЭТФ. 2002. Т. 76. В. 11. С. 779-783.

[15] Kittel C. // Phys. Rev. 1958. V. 110. P. 1295-1297.

[16] Morales M.A., Lassri H., Biondo A., Rossi A.M., BaggioSaitovitch E. // J. Magn. Magn. Mater. 2003. V. 256. P. 93-99. https://doi.org/10.1016/S0304-8853(02)00386-4

[17] Poimanov V.D., Kuchko A.N., Kruglyak V.V. // Phys. Rev. B. 2018. V. 98. P. 104418.

[18] Исхаков Р.С., Яковчук В.Ю., Столяр С.В., Чеканова Л.А., Середкин B.A. // ФТТ. 2001. Т. 43. В. 8. С. 1462-1466. https://journals.ioffe.ru/articles/38276

[19] Исхаков Р.С., Шепета Н.А., Комогориев С.В., Столяр С.В., Чеканова Л.А., Бондаренко Г.Н., Мальцев В.К., Балаев А.Д. // ФММ. 2003. Т. 95. № 3. С. 37-42. 